Gut and Liver, Vol. 10, No. 4, July 2016, pp. 526-531

\title{
Comparison of AIMS65 Score and Other Scoring Systems for Predicting Clinical Outcomes in Koreans with Nonvariceal Upper Gastrointestinal Bleeding
}

Sung Min Park, Seok Cheon Yeum, Byung-Wook Kim, Joon Sung Kim, Ji Hee Kim, Eun Hui Sim, Jeong-Seon Ji, and Hwang Choi

Division of Gastroenterology, Department of Internal Medicine, Incheon St. Mary's Hospital, The Catholic University of Korea College of Medicine, Incheon, Korea

Background/Aims: The AIMS65 score has not been sufficiently validated in Korea. The objective of this study was to compare the AIMS65 and other scoring systems for the prediction of various clinical outcomes in Korean patients with acute nonvariceal upper gastrointestinal bleeding (NVUGIB). Methods: The AIMS65 score, clinical and full Rockall scores (cRS and fRS) and Glasgow-Blatchford (GBS) score were calculated in patients with NVUGIB in a single center retrospectively. The performance of these scores for predicting mortality, rebleeding, transfusion requirement, and endoscopic intervention was assessed by calculating the area under the receiver-operating characteristic curve. Results: Of the 523 patients, $3.4 \%$ died within 30 days, $2.5 \%$ experienced rebleeding, $40.0 \%$ required endoscopic intervention, and $75.7 \%$ needed transfusion. The AIMS65 score was useful for predicting the 30-day mortality, the need for endoscopic intervention and for transfusion. The fRS was superior to the AIMS65, GBS, and cRS for predicting endoscopic intervention and the GBS was superior to the AIMS65, fRS, and cRS for predicting the transfusion requirement. Conclusions: The AIMS65 score was useful for predicting the 30-day mortality, transfusion requirement, and endoscopic intervention in Korean patients with acute NVUGIB. However, it was inferior to the GBS and fRS for predicting the transfusion requirement and endoscopic intervention, respectively. (Gut Liver 2016;10:526-531)

Key Words: Nonvariceal upper gastrointestinal bleeding; AIMS65 score; Rockall score; Glasgow-Blatchford score

\section{INTRODUCTION}

Upper gastrointestinal bleeding (UGIB) is a common medical emergency, with an incidence of approximately 160 in 100,000 people per year. ${ }^{1}$ The main cause of UGIB is peptic ulcer disease, which accounts for $20 \%$ to $50 \%$ of all cases. ${ }^{2,3}$ Patients with nonvariceal UGIB (NVUGIB) may present with a wide range of clinical severity, ranging from minor bleeding to death. The American College of Gastroenterology practice guidelines and International Consensus guidelines recommend stratifying patients with UGIB into high- and low-risk categories through risk assessments. ${ }^{4,5}$ Several risk scoring systems have been introduced to differentiate high- and low-risk patients. The Rockall score and the Glasgow-Blatchford risk score (GBS) are the best known and most widely used scoring systems. Several studies have compared GBS and Rockall scores in the prediction of several clinically significant outcomes, ${ }^{6-13}$ but it is not clear which of these two is superior. Neither score has been adopted in routine clinical practice because each presents limitations. Specifically, the Rockall score is not suitable for pre-endoscopic triage due to the need for endoscopic assessment, and GBS is complex to calculate and contains some variables that lack a clear definition.

Recently, AIMS 65, a novel scoring system that consists of five parameters, i.e., albumin levels, prothrombin time international normalized ration, altered mental status, systolic blood pressure, and age, was designed to predict inpatient mortality in patients with UGIB by using data that are easily calculated and routinely obtained in the emergency department. ${ }^{14}$ The AIMS65 score has been useful to predict several clinical outcomes in

Correspondence to: Byung-Wook Kim

Division of Gastroenterology, Department of Internal Medicine, Incheon St. Mary's Hospital, The Catholic University of Korea College of Medicine, 56 Dongsu-ro, Bupyeong-gu, Incheon 21431, Korea

Tel: +82-32-280-5051, Fax: +82-32-280-5987, E-mail: gastro@catholic.ac.kr Received on March 27, 2015. Revised on May 14, 2015. Accepted on July 29, 2015. pISSN 1976-2283 eISSN 2005-1212 http://dx.doi.org/10.5009/gnl15153

Sung Min Park and Seok Cheon Yeum contributed equally to this work as first authors.

@ This is an Open Access article distributed under the terms of the Creative Commons Attribution Non-Commercial License (http://creativecommons.org/licenses/by-nc/4.0) which permits unrestricted non-commercial use, distribution, and reproduction in any medium, provided the original work is properly cited. 
Western studies. ${ }^{14,15}$ However, it has been validated for Korean patients in one study only and was insufficient for predicting poor outcomes. ${ }^{16}$ Further, only a few studies have compared AIMS65 score with other scoring systems ${ }^{15,17}$ and validated the use of the AIMS65 score in patients with NVUGIB.

Therefore, the first aim of this study was to validate AIMS65 score for the prediction of 30-day mortality, rebleeding, transfusion requirement and endoscopic intervention in Korean patients with acute NVUGIB. The second aim was to compare AIMS65 with other scoring systems including clinical Rockall score, full Rockall score, and GBS.

\section{MATERIALS AND METHODS}

\section{Data collection and outcomes verification}

We obtained data retrospectively from consecutive patients who were suspected UGIB due to hematemesis, melena or decreased hemoglobin level in Incheon St. Mary's Hospital, The Catholic University of Korea. Between January 1, 2009 and December 31, 2013, a total of 5,601 consecutive patients were collected and reviewed. The exclusion criteria were as follows: (1) did not undergo endoscopy; (2) no evidence of bleeding in endoscopy; (3) variceal bleeding; (4) younger than 18 years old; (5) bleeding from iatrogenic lesions such as after endoscopic resection; (6) could not obtain at least one of the four scoring systems; and (7) followed up for fewer than 30 days. Admission history, clinical and laboratory data, endoscopic findings, treatment and clinical follow-up were investigated based on four scoring systems (clinical Rockall score, full Rockall score, GBS, and AIMS65 score). To calculate the values of each scoring systems, the following data were collected through manual chart review: age, sex, comorbidity, presence of melena or syncope, altered mental status, systolic blood pressure, heart rate, hemoglobin, serum albumin, international normalized ratio, blood urea nitrogen, endoscopic findings, and total pack of transfusion. Patients who developed an UGIB while hospitalized for an unrelated disease were also included. This study protocol was approved by the Institutional Review Board of Incheon St. Mary's Hospital, The Catholic University of Korea (OC14RISI0012).

Using receiver-operating characteristic curves, we compared the four scoring systems in predicting 30-day mortality, rebleeding, transfusion requirements, and endoscopic interventions. The 30-day mortality included deaths that were either unrelated or related to UGIB. Rebleeding was defined as repeated endoscopy due to unstable vital signs, with a decreased hemoglobin concentration of at least $2.0 \mathrm{~g} / \mathrm{dL}$ per day before hospital discharge, surgery or arterial embolization for control of UGIB or readmission to the hospital within 30 days of discharge due to UGIB. The data for each scoring system were entered by research nurses who were blinded to the final outcomes, and each system was scored by one gastroenterologist (S.C.Y.), according to the scoring system reported by the authors of the original articles.

\section{Statistical analysis}

The area under the receiver-operating characteristic curve (AUROC) was calculated for each score with confidence intervals. AUROCs were tested for equality using the Delong test. Sensitivity and specificity in detecting patients who had died within 30 days, recurrent bleeding, transfusion requirement, or need for endoscopic intervention were calculated for the clinical and full Rockall scores, GBS, and AIMS65 score with 95\% confidence intervals. A p-value of less than 0.05 was considered statistically significant. The data analysis was performed using the SPSS statistical software version 19.0 (SPSS Inc., Chicago, IL, USA).

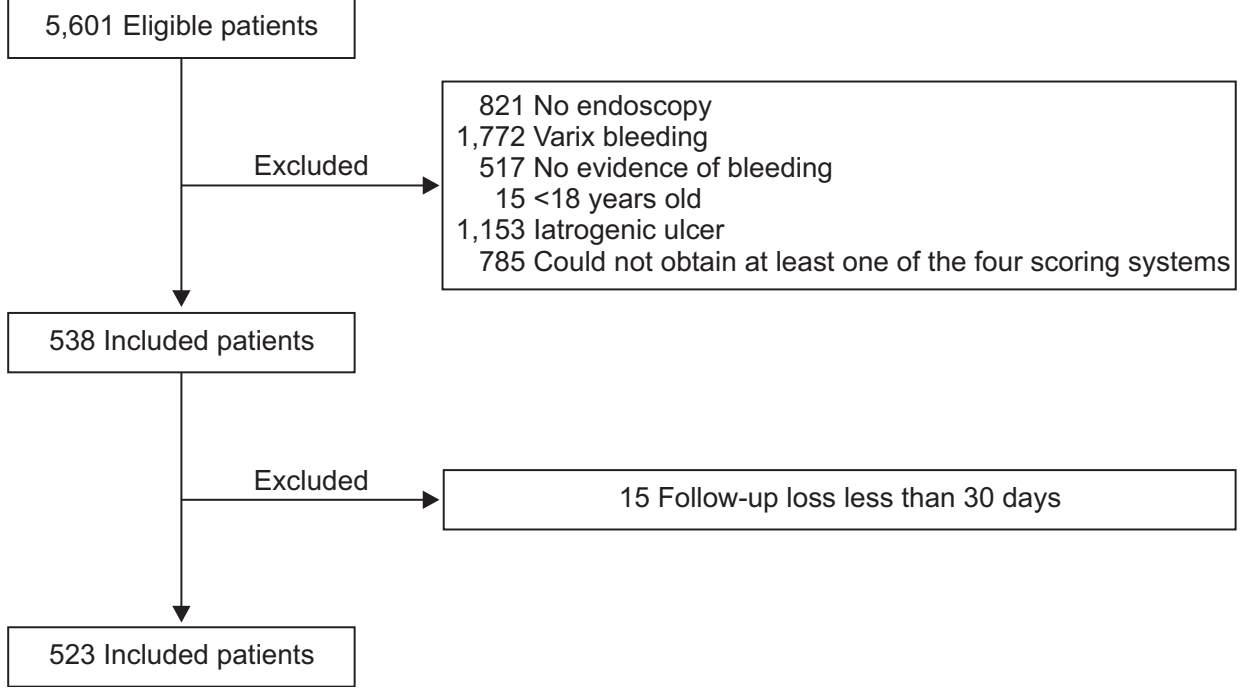

Fig. 1. Flow chart of patients enrolled in this study. 


\section{RESULTS}

\section{Patient characteristics}

Among the 5,601 consecutive patients who received intravenous proton pump inhibitor therapy, 5,078 patients were excluded, according to the exclusion criteria. A total of 523 patients were enrolled (Fig. 1).

The basic characteristics of the patients and their clinical outcomes are described in Table 1. Of the 523 patients, 369 patients (70.6\%) were men, and 154 patients (29.4\%) were women. The patients' mean age was $59.9 \pm 15.0$ years. On initial esophagogastroduodenoscopy, 420 patients (80.3\%) had peptic ulcer bleeding (gastric ulcer bleeding in 279 patients [53.3\%] and duodenal ulcer bleeding in 141 patients [27.0\%]). Additionally, 47 patients (9.0\%) were diagnosed with Mallory-Weiss tearing, 30 patients (5.7\%) had gastric cancer bleeding and 26 patients (5.0\%) showed other diseases (e.g., Dieulafoy's lesion, esophageal ulcer, acute gastric mucosal lesion, diverticular bleeding, and angiodysplasia).

\section{Clinical outcomes}

A total of 18 patients (3.4\%) died, five of whom died due to causes associated with bleeding. The 13 patients of those died of septic shock in three patients, renal failure in two patients, neoplasm in three patients, hospital-acquired pneumonia in three patients and liver failure in two patients. Additionally, 13 patients (2.5\%) experienced rebleeding, and 209 (40.0\%) and 396 patients $(75.7 \%)$ received transfusion and endoscopic intervention, respectively.

\section{Thirty-day mortality}

For the prediction of 30-day mortality, the AUROC was 0.79 for the AIMS65 score (95\% confidence interval [CI], 0.69 to 0.88 ; $\mathrm{p}=0.000$ ), 0.76 for the clinical Rockall score ( $95 \%$ CI, 0.67 to 0.86 ; $\mathrm{p}=0.000$ ), 0.81 for the full Rockall score ( $95 \% \mathrm{CI}, 0.73$ to 0.89 ; $\mathrm{p}=0.000$ ) and 0.61 for GBS (95\% CI, 0.49 to 0.88 ; $p=0.114$ ). The AIMS65, clinical Rockall score and full Rockall score all predicted 30-day mortality well, without any significant difference among the scores. The AUROCs of the four scores for predicting 30-day mortality, rebleeding, endoscopic treatment and transfusion are shown in Fig. 2, and the comparisons of the AUROCs are summarized in Table 2.

\section{Rebleeding}

For the prediction of rebleeding, the AUROC was 0.61 for the AIMS65 score (95\% CI, 0.44 to $0.78 ; p=0.164$ ), 0.72 for the clinical Rockall score (95\% CI, 0.59 to $0.85 ; p=0.008$ ), 0.77 for the full Rockall score (95\% CI, 0.65 to $0.88 ; p=0.001$ ) and 0.71 for GBS (95\% CI, 0.59 to 0.83 ; $\mathrm{p}=0.009$ ). The clinical Rockall score, full Rockall score and GBS were useful for predicting rebleeding, and the ability to predict rebleeding was equal for the 3 scores.
Table 1. Basic Characteristics and Clinical Outcomes of the Study Population

\begin{tabular}{|c|c|}
\hline Characteristic & Value \\
\hline Age, yr & $59.9 \pm 15.0$ \\
\hline \multicolumn{2}{|l|}{ Sex } \\
\hline Male & 369 (70.6) \\
\hline Female & $154(29.4)$ \\
\hline \multicolumn{2}{|l|}{ Comorbidity } \\
\hline None & $400(74.3)$ \\
\hline Liver cirrhosis & $34(6.3)$ \\
\hline Heart failure & $5(0.9)$ \\
\hline Ischemic heart disease & $33(6.1)$ \\
\hline Renal failure & $50(9.3)$ \\
\hline Metastatic cancer & $26(4.8)$ \\
\hline \multicolumn{2}{|l|}{ Lab variables } \\
\hline Hemoglobin & $8.72 \pm 2.65$ \\
\hline Blood urea nitrogen & $38.9 \pm 26.6$ \\
\hline International normalized ratio & $1.21 \pm 0.64$ \\
\hline Albumin & $3.45 \pm 0.60$ \\
\hline \multicolumn{2}{|l|}{ Clinical variables } \\
\hline Melena & $388(74.2)$ \\
\hline Hematemesis & $157(30.0)$ \\
\hline Shock & $114(21.8)$ \\
\hline Tachycardia & $193(36.9)$ \\
\hline Syncope & $21(3.9)$ \\
\hline Decreased mentality & $14(2.7)$ \\
\hline \multicolumn{2}{|l|}{ Score } \\
\hline Clinical Rockall & $2(0-7)$ \\
\hline Full Rockall & $4(0-10)$ \\
\hline Glasgow-Blatchford & $13(2-21)$ \\
\hline AIMS65 & $1(0-4)$ \\
\hline \multicolumn{2}{|l|}{ Source of acute NVUGIB } \\
\hline Gastric ulcer & $279(53.3)$ \\
\hline Duodenal ulcer & $141(27.0)$ \\
\hline Mallory Weiss tear & $47(9.0)$ \\
\hline Malignancy & $30(5.7)$ \\
\hline Dieulafoy's lesion & $11(2.1)$ \\
\hline Esophageal ulcer & $11(2.1)$ \\
\hline Others & $4(0.8)$ \\
\hline \multicolumn{2}{|l|}{ Clinical outcomes } \\
\hline 30-Day mortality & $18(3.4)$ \\
\hline Rebleeding & $13(2.5)$ \\
\hline Transfusion requirement & $209(40.0)$ \\
\hline Endoscopic intervention & $396(75.7)$ \\
\hline
\end{tabular}

Data are presented as mean $\pm \mathrm{SD}$, number (\%), or median (range). The sum of rates of each variable in comorbidity and clinical variables were more than $100 \%$ due to overlap. NVUGIB, nonvariceal upper gastrointestinal bleeding. 
A

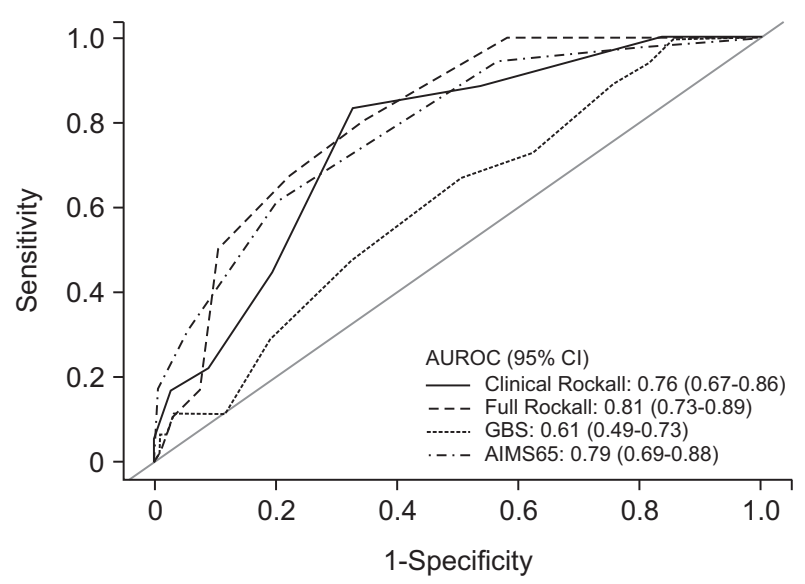

C

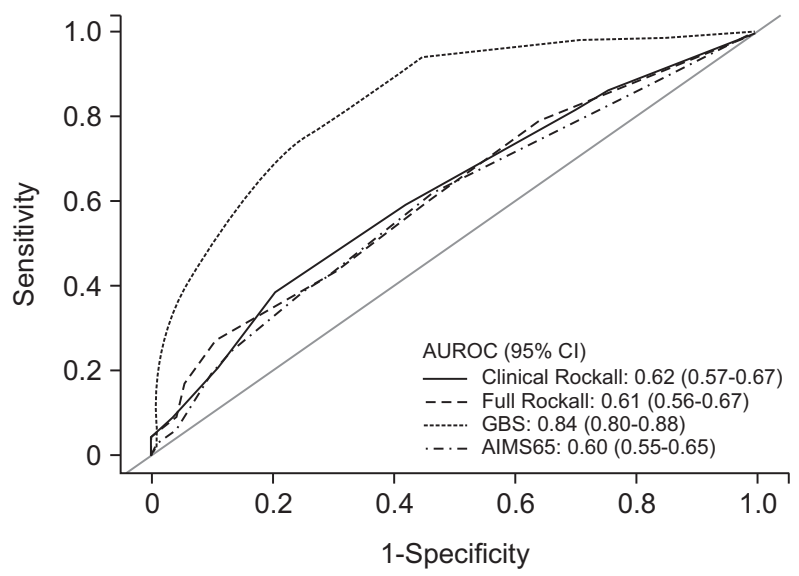

B

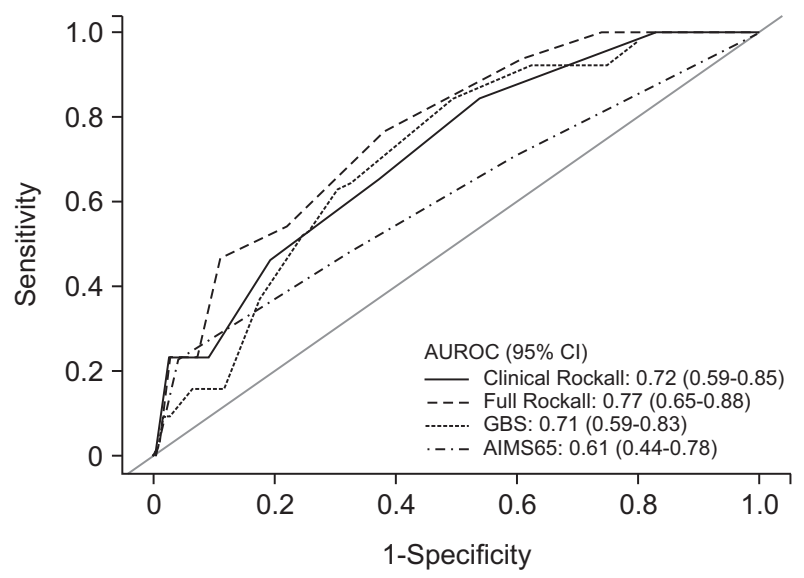

D

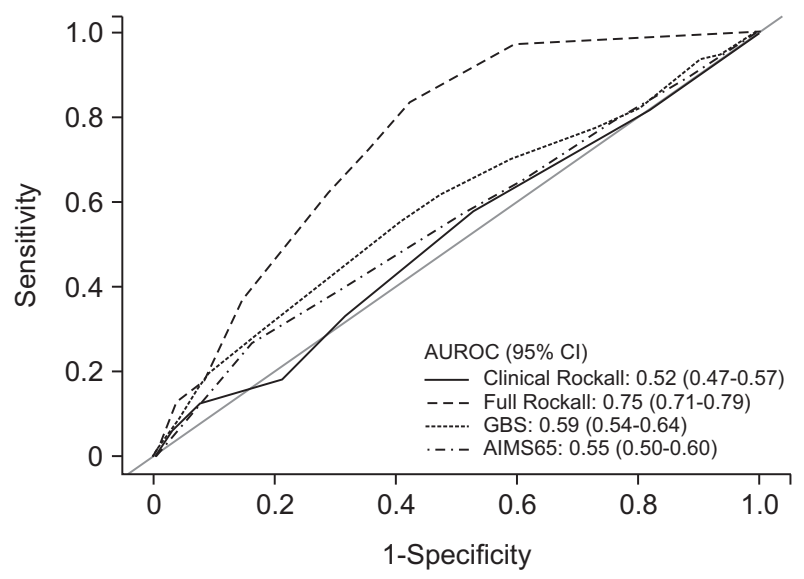

Fig. 2. Comparisons of the clinical Rockall, full Rockall, GBS, and AIMS65 scores with AUROC curves for the prediction of 30-day mortality (A), rebleeding (B), transfusion requirements (C), and endoscopic intervention (D).

GBS, Glasgow Blatchford score; AUROC, area under the receiver-operating characteristic curve.

\section{Transfusion requirements}

For the prediction of transfusion requirements, the AUROC was 0.60 for the AIMS65 score (95\% CI, 0.55 to $0.65 ; \mathrm{p}=0.001$ ), 0.62 for the clinical Rockall score (95\% CI, 0.57 to 0.67 ; $\mathrm{p}=0.000$ ), 0.61 for the full Rockall score ( $95 \% \mathrm{CI}, 0.56$ to 0.67 ; $\mathrm{p}=0.000$ ) and 0.84 for GBS (95\% CI, 0.80 to 0.88 ; $\mathrm{p}=0.000$ ). Although all four scoring systems predicted the need for transfusion well, GBS was superior to the the AIMS65, full Rockall, and clinical Rockall score in predicting transfusion requirement $(\mathrm{p}<0.0001, \mathrm{p}<0.0001$, and $\mathrm{p}<0.0001$, respectively).

\section{Endoscopic intervention}

For the need of endoscopic intervention, the AUROC was 0.55 for the AIMS65 score (95\% CI, 0.50 to $0.60 ; p=0.050$ ), 0.52 for the clinical Rockall score ( $95 \%$ CI, 0.47 to 0.57 ; $p=0.509$ ), 0.75 for the full Rockall score (95\% CI, 0.71 to $0.79 ; \mathrm{p}=0.000$ ), and 0.59 for GBS (95\% CI, 0.54 to $0.64 ; \mathrm{p}=0.001$ ). Although all of the scores but the clinical Rockall score were useful for predict- ing the need of endoscopic intervention, the full Rockall score was superior to the AIMS65, GBS and clinical Rockall score in predicting endoscopic intervention $(\mathrm{p}<0.0001, \mathrm{p}<0.0001$, and $\mathrm{p}<0.0001$, respectively).

\section{DISCUSSION}

In our study, the AIMS65 score was able to predict 30-day mortality, transfusion requirement and endoscopic intervention, except rebleeding, in NVUGIB patients. The AIMS65 score was similar to the clinical Rockall and full Rockall scores in predicting 30-day mortality. Although the AIMS65 score was useful for predicting the transfusion requirement and endoscopic intervention, the GBS and full Rockall score were superior to other scores, including the AIMS65 score, in predicting transfusion requirement and endoscopic intervention, respectively.

The AIMS65 score was developed to predict in-hospital mortality in UGIB. ${ }^{14}$ It consists of five parameters that can be obtained simply in the emergency department and can then 
Table 2. Comparison of AUROC for the Four Scoring Systems

\begin{tabular}{|c|c|c|c|c|c|}
\hline & \multirow{2}{*}{ AUROC (95\% CI) } & \multicolumn{4}{|c|}{ p-value for compare the AUROC curves } \\
\hline & & AIMS65 & Clinical Rockall & Full Rockall & GBS \\
\hline \multicolumn{6}{|l|}{ 30-Day mortality } \\
\hline AIMS65 & 0.79 (0.69-0.88) & - & 0.7050 & 0.6627 & 0.0408 \\
\hline Clinical Rockall & $0.76(0.67-0.86)$ & - & - & 0.0788 & 0.0249 \\
\hline Full Rockall & $0.81(0.73-0.89)$ & - & - & - & 0.0027 \\
\hline GBS & $0.61(0.49-0.73)$ & - & - & - & - \\
\hline \multicolumn{6}{|l|}{ Rebleeding } \\
\hline AIMS65 & $0.61(0.44-0.78)$ & - & 0.2673 & 0.0478 & 0.3869 \\
\hline Clinical Rockall & $0.72(0.59-0.85)$ & - & & 0.2282 & 0.9598 \\
\hline Full Rockall & $0.77(0.65-0.88)$ & - & - & - & 0.5031 \\
\hline GBS & $0.71(0.59-0.83)$ & - & - & - & - \\
\hline \multicolumn{6}{|c|}{ Transfusion requirement } \\
\hline AIMS65 & $0.60(0.55-0.65)$ & - & 0.4178 & 0.5662 & $<0.0001$ \\
\hline Clinical Rockall & $0.62(0.57-0.67)$ & - & - & 0.7283 & $<0.0001$ \\
\hline Full Rockall & $0.61(0.56-0.67)$ & - & - & - & $<0.0001$ \\
\hline GBS & $0.84(0.80-0.88)$ & - & - & - & - \\
\hline \multicolumn{6}{|c|}{ Endoscopic intervention } \\
\hline AIMS65 & $0.55(0.50-0.60)$ & - & 0.1866 & $<0.0001$ & 0.2067 \\
\hline Clinical Rockall & $0.52(0.47-0.57)$ & - & - & $<0.0001$ & 0.0089 \\
\hline Full Rockall & $0.75(0.71-0.79)$ & - & - & - & $<0.0001$ \\
\hline GBS & $0.59(0.54-0.64)$ & - & - & - & - \\
\hline
\end{tabular}

AUROC, area under the receiver-operating characteristic curve; CI, confidence interval; GBS, Glasgow Blatchford score.

be easily adopted to stratify patients with UGIB into high- and low-risk groups in clinical practice. However, the AIMS65 score has only been validated in a few studies, ${ }^{14-17}$ and further extensive validation is needed. Therefore, we validated the AIMS65 score for predicting four clinical outcomes and compared it with the three best-known risk scores.

According to previous studies, ${ }^{14,15}$ the AIMS65 has a good predictive ability for inpatient mortality in patients with UGIB. In accordance with previous studies, ${ }^{14,15}$ the AIMS65 score was useful for predicting 30-day mortality in patients with NVUGIB in the present study. A report showed that the AIMS65 score had poor predictive ability for a composite clinical outcome of rebleeding and mortality in patients with peptic ulcer bleeding. ${ }^{16}$ However, their study only contained one patient who died within 30 days, among the total of 149 patients with peptic ulcer bleeding. Therefore, further studies are needed to validate the AIMS65 score for predicting mortality in peptic ulcer bleeding patients. Additionally, the AIMS65 was shown to be able to predict several clinical outcomes. ${ }^{15}$ In the present study, the AIMS65 score was useful for predicting transfusion requirement and endoscopic intervention, except rebleeding. Therefore, the AIMS65 score might be used to predict clinical interventions and mortality.
According to a recent study, ${ }^{15}$ the AIMS65 score was superior to the GBS in predicting inpatient mortality but inferior to the GBS in predicting the requirement of transfusion; that was consistent with the results of the present study. However, the AIMS65 score was not useful for predicting rebleeding in the present study. Although the causes of the different results for rebleeding from the previous study are unclear, the differences in ethnicity and cause of UGIB might be the reasons. To the best of our knowledge, the AIMS65 score has never been compared with the clinical or full Rockall scores for predicting clinical outcomes. This study was the first to compare the AIMS65 score and the Rockall scores. The AIMS65 score was similar to the clinical and full Rockall scores in predicting 30-day mortality and transfusion requirement but was inferior to clinical and full Rockall scores in predicting rebleeding. The AIMS65 score was inferior to the full Rockall score in predicting endoscopic intervention. These results might be caused by the inclusion of endoscopic variables in the full Rockall score.

Our study has some limitations. First, this study was a singlecenter study with a cohort consisting of only Korean patients. The results of this study may not be generalized to other countries and medical institutions. Second, this study has the limitations associated with a retrospective design. Third, variation for 
the choice of endoscopic intervention might exist between endoscopists, since it was at discretion of individual endoscopist.

In conclusion, the AIMS65 score was useful as the other scoring systems for predicting short term morality in Korean patients with NVUGIB. However, the AIMS 65 was inferior to the other scoring systems in predicting clinical intervention. Considering that the present study was retrospective one, further prospective studies validating the AIMS 65 score for diverse clinical outcomes and comparing AIMS65 with other scoring systems in various populations are anticipated. Also, new scoring system may be necessary, because no single scoring system seem to be superior to others in predicting outcomes in the present study.

\section{CONFLICTS OF INTEREST}

No potential conflict of interest relevant to this article was reported.

\section{REFERENCES}

1. Lewis JD, Bilker WB, Brensinger C, Farrar JT, Strom BL. Hospitalization and mortality rates from peptic ulcer disease and GI bleeding in the 1990s: relationship to sales of nonsteroidal antiinflammatory drugs and acid suppression medications. Am J Gastroenterol 2002;97:2540-2549.

2. Jutabha R, Jensen DM. Management of upper gastrointestinal bleeding in the patient with chronic liver disease. Med Clin North Am 1996;80:1035-1068.

3. Boonpongmanee S, Fleischer DE, Pezzullo JC, et al. The frequency of peptic ulcer as a cause of upper-GI bleeding is exaggerated. Gastrointest Endosc 2004;59:788-794.

4. Laine L, Jensen DM. Management of patients with ulcer bleeding. Am J Gastroenterol 2012;107:345-360.

5. Barkun AN, Bardou M, Kuipers EJ, et al. International consensus recommendations on the management of patients with nonvariceal upper gastrointestinal bleeding. Ann Intern Med 2010;152:101113.

6. Laursen SB, Hansen JM, Schaffalitzky de Muckadell OB. The Glasgow Blatchford score is the most accurate assessment of patients with upper gastrointestinal hemorrhage. Clin Gastroenterol Hepatol 2012;10:1130-1135.e1.
7. Chen IC, Hung MS, Chiu TF, Chen JC, Hsiao CT. Risk scoring systems to predict need for clinical intervention for patients with nonvariceal upper gastrointestinal tract bleeding. Am J Emerg Med 2007;25:774-779.

8. Stanley AJ, Dalton HR, Blatchford 0, et al. Multicentre comparison of the Glasgow Blatchford and Rockall Scores in the prediction of clinical end-points after upper gastrointestinal haemorrhage. Aliment Pharmacol Ther 2011;34:470-475.

9. Pang SH, Ching JY, Lau JY, Sung JJ, Graham DY, Chan FK. Comparing the Blatchford and pre-endoscopic Rockall score in predicting the need for endoscopic therapy in patients with upper GI hemorrhage. Gastrointest Endosc 2010;71:1134-1140.

10. Stanley AJ, Ashley D, Dalton HR, et al. Outpatient management of patients with low-risk upper-gastrointestinal haemorrhage: multicentre validation and prospective evaluation. Lancet 2009;373:4247.

11. Bryant RV, Kuo P, Williamson K, et al. Performance of the Glasgow-Blatchford score in predicting clinical outcomes and intervention in hospitalized patients with upper GI bleeding. Gastrointest Endosc 2013;78:576-583.

12. Gralnek IM, Dulai GS. Incremental value of upper endoscopy for triage of patients with acute non-variceal upper-GI hemorrhage. Gastrointest Endosc 2004;60:9-14.

13. Chandra S, Hess EP, Agarwal D, et al. External validation of the Glasgow-Blatchford bleeding score and the Rockall score in the US setting. Am J Emerg Med 2012;30:673-679.

14. Saltzman JR, Tabak YP, Hyett BH, Sun X, Travis AC, Johannes RS. A simple risk score accurately predicts in-hospital mortality, length of stay, and cost in acute upper GI bleeding. Gastrointest Endosc 2011;74:1215-1224.

15. Hyett BH, Abougergi MS, Charpentier JP, et al. The AIMS65 score compared with the Glasgow-Blatchford score in predicting outcomes in upper GI bleeding. Gastrointest Endosc 2013;77:551557.

16. Jung SH, Oh JH, Lee HY, et al. Is the AIMS65 score useful in predicting outcomes in peptic ulcer bleeding? World J Gastroenterol 2014;20:1846-1851.

17. Yaka E, Yılmaz S, Doğan NÖ, Pekdemir M. Comparison of the Glasgow-Blatchford and AIMS65 scoring systems for risk stratification in upper gastrointestinal bleeding in the emergency department. Acad Emerg Med 2015;22:22-30. 